\title{
Preliminary Study on the Effectiveness of Different Durations of Hot Towel Application to the Back during Bed Bathing
}

\author{
Inaho Shishido', Yuka Yamaguchi², Rie Miyata ${ }^{3}$, Sakiko Kutomi ${ }^{4}$, Rika Yano $^{{ }^{*}}$ \\ ${ }^{1}$ KKR Medical Center, Sapporo, Japan \\ ${ }^{2}$ The University of Tokyo Hospital, Tokyo, Japan \\ ${ }^{3}$ Elderly Support Section, Fukagawa City, Fukagawa, Japan \\ ${ }^{4}$ Toranomon Hospital, Tokyo, Japan \\ ${ }^{5}$ Faculty of Health Sciences, Hokkaido University, Sapporo, Japan \\ Email: *r-yano@med.hokudai.ac.jp
}

How to cite this paper: Shishido, I., Yamaguchi, Y., Miyata, R., Kutomi, S. and Yano, R. (2017) Preliminary Study on the Effectiveness of Different Durations of Hot Towel Application to the Back during Bed Bathing. Open Journal of Nursing, 7, 1375-1386.

https://doi.org/10.4236/ojn.2017.712099

Received: November 10, 2017

Accepted: December 8, 2017

Published: December 11, 2017

Copyright (C) 2017 by authors and Scientific Research Publishing Inc. This work is licensed under the Creative Commons Attribution International License (CC BY 4.0).

http://creativecommons.org/licenses/by/4.0/

\begin{abstract}
The purpose of this study was to determine the most effective duration of hot towel application during bed bathing, based on temporal changes in skin surface temperature, towel temperature, and subjective comfort. As a secondary objective, differences in skin surface temperature and subjective comfort for hot towel application on the back and dry towel wiping alone were evaluated. For the first objective, hot towels were applied on the lower back of 20 healthy adults, for different duration $(10,15$, and $20 \mathrm{~s})$, with a 10 -s duration found to be the most effective. For the second objective, we compared bed bathing with hot towel application to dry wiping alone, $\mathrm{n} 21$ healthy adult participants. A 10-s hot towel application increased the surface temperature of the skin $\left(+0.5^{\circ} \mathrm{C}\right)$ and provided a perceived sensation of warmth and comfort. In contrast, dry wiping significantly decreased the surface skin temperature $\left(-0.8^{\circ} \mathrm{C}\right)$. In conclusion, hot towel application increased skin surface temperature and improved subjective warmth and comfort during bed bathing.
\end{abstract}

\section{Keywords}

Bed Bath, Hot Towel Application, Skin Surface Temperature

\section{Introduction}

Bed baths are an important component of nursing care to keep the skin clear, as well as to refresh a patient and enhance circulation, and provide a sensation of well-being [1]. Various bed bathing techniques have been developed. In the ab- 
sence of comparative evidence of effectiveness, nurses must select best practice on their own. The selected application of hot towels during bed bathing is a specific area of controversy in practice.

In Japan, some nurses attempt to provide warmth and comfort by applying hot towels to their patient's skin during bed bathing. Shishido and Yano [2] reported that a 10-s application of a hot towel to the palmar surface of the forearm in elderly individuals maintained skin barrier function, as well as providing "warmth" and a "very pleasant". Without this application, the temperature of the surface of the skin was lower and the skin barrier function impaired. In practice, hot towels are typically applied to the lower back, an area very sensitive to temperature sensation due to the densely packed warm and cool receptor areas in this region [3]. Application of a hot towel to the lumbar region may, therefore, increase skin surface temperature and improve circulation, in addition to providing a pleasant "sensation" and "relaxation" [4] [5] [6]. However, reports of the effectiveness of this practice are limited to Japanese-language studies.

Among studies from Japan, variable hot towel preparation methods have been reported, with different techniques of application and duration. Of these issues, the duration of application requires specific assessment of effectiveness as a brief duration may cause a decrease in towel temperature through heat evaporation. However, the relationship between perceive comfort and the temporal change in both skin and towel temperature has not yet been clarified and the most effective duration for application of a hot towel remains unclear for practice. Therefore, the aim of our study was to determine the most effective duration of hot towel application to the back during bed bathing, based on temporal changes in skin surface temperature, towel temperature, and subjective comfort. As a secondary objective, we also evaluated differences in skin surface temperature and subjective comfort for hot towel application on the back and dry towel wiping only.

\section{Methods and Materials}

\subsection{Participants}

Two study protocols were used to address the primary and secondary objectives of the study. For the first objective of determining the most effective duration of hot towel application to the back during bed bathing (Experiment A). It was thought that the experience of bed bath affected the experiment result; thus, we recruited 20 healthy university students (10 men and 10 women), $\geq 20$-years-old, who had no prior experience with bed bathing. Eligible participants were recruited from a national university via posters and flyers and agreed to participate in the study. All subjects did not experience the Bed bath during experimental period. For the second objective of evaluating differences in skin surface temperature and subjective comfort for hot towel application (AHT 10-s) on the back and dry towel wiping only, we recruited 20 healthy students (11 men and 10 women) university students, $\geq 21$-years-old, who had no prior experience with bed bathing. For both experiments, participants were encouraged to have a 
sufficient amount of sleep on the night prior to the experiment, and to refrain from consuming alcohol within $8 \mathrm{~h}$ prior to the experiment. On the day of the experiment, participants were asked to refrain from eating spicy food or drinking large quantities of caffeinated beverages, performing strenuous exercise that might cause sweating, and to stop eating $1 \mathrm{~h}$ before the experiment.

The sample size was confirmed using $G^{*}$ Power software (ver. 3.1.9), with 14 participants calculated as the minimum sample size to detect a difference using paired $t$-test analysis, with a level of significance of 0.05 , power of 0.80 and effect size of 0.50 , based the author's prior research [2]. Considering the possibility of dropout, 20 individuals were recruited for each experiment, A and B.

\subsection{Statement of Ethics}

The aims and experimental methods were explained to participants, including the right to withdraw from the study at any point without prejudice and insurance that anonymity would be protected throughout all components of the study. All participants provided informed consent. Our study protocols were approved by the ethics committee of the Hokkaido University Faculty of Health Sciences (approval no. 14-14).

\subsection{Protocol for Experiment A}

Participants were placed in sitting, leaning forward to lightly hug a pillow placed on over-bed table, adjusted to each participant's preferred height (Figure 1). A cloth $(74 \times 35 \mathrm{~cm}, 82.0 \pm 2.0 \mathrm{~g})$ was placed in a thermostatically controlled bath (TR-2AR Thermal Robo; As One, Osaka, Japan) filled with water at a temperature of $50^{\circ} \mathrm{C} \pm 0.02^{\circ} \mathrm{C}$. After heating, excess water was wrung from the cloth, with a final weight of $240.0 \pm 5.0 \mathrm{~g}$. The hot towel was immediately applied to

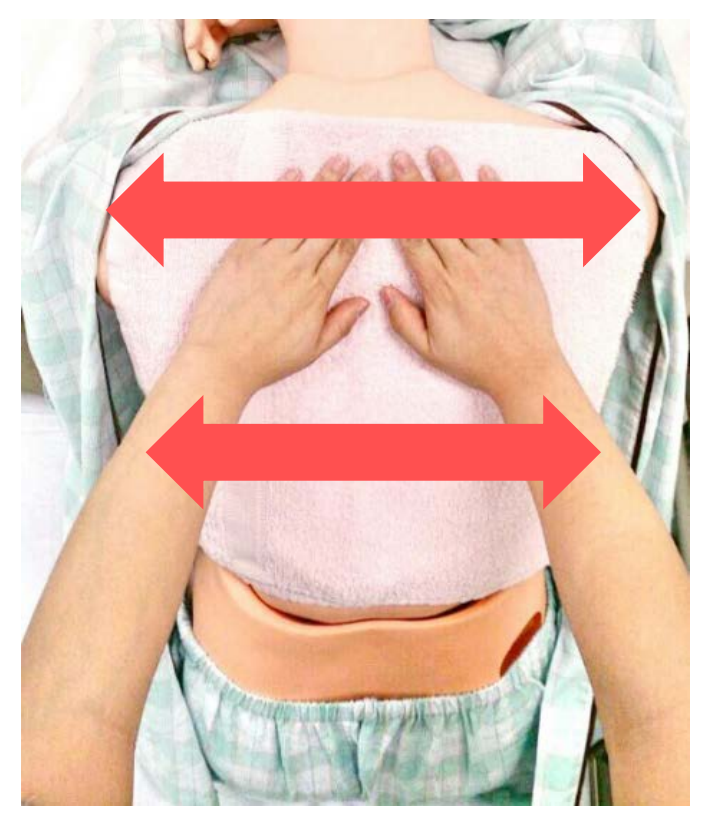

Figure 1. Application of the hot towel on back. 
participants' back, with the upper edge of the cloth along placed a line connecting the right and left acromion. Two previous studies have reported that a 10-s application of a hot towel (AHT 10-s) to the palmar surface of the forearm for both young and elderly individuals was sufficient to significantly increase skin surface temperature, by $2.3^{\circ} \mathrm{C}-3.6^{\circ} \mathrm{C}$, and to improve subjective sensations of warmth and comfort [7] [8]. As the back area is a wider area, we evaluated the effectiveness of 3 different durations of AHT, 10, 15 and 20 s (AHT 10-s, AHT 15-s and AHT 20-s, respectively), with the order of application randomly selected across participants.

A crossover study design was used (Figure 2), with all experiment conducted within a 6-h window, between 10:00 and 16:00 $\mathrm{H}$, when autonomic function is deemed to be stable [9]. To standardize the experimental conditions, measurements were taken in a room with a controlled temperature $\left(20^{\circ} \mathrm{C}-22^{\circ} \mathrm{C}\right)$ and humidity $(40 \%-60 \%)$ [10] [11], with the same researcher applying the hot towel and wiping the skin for all participants.

Upon entering the room, participants changed into a hospital gown, and their height and weight was measured. After a 10-min rest period, a still thermographic image was taken of the surface temperature of the skin of the back. The hot towel was then placed on a participant's back, with the researcher applying constant pressure with both hands on the hot towel, from the center to the outer edges of the towel, for $10 \mathrm{~s}$. The same procedure was repeated for the 15 and $20 \mathrm{~s}$ application. A 15-min rest period was provided between applications, with the order of duration randomizes a priori, with confirmation that the skin surface temperature of the back had returned to baseline value between applications.

During the application, a still thermography image of the surface temperature of the exterior surface (exposed to the air) of the towel was obtained. The towel was removed according to the pre-set application duration, and thermographic images were immediately obtained of the skin surface and the interior surface of the towel (touching the skin). The back was wiped dry and another image of skin surface temperature obtained. During the application, participants completed a questionnaire regarding warmth, comfort and duration of application. If a participant complained of excessive skin heating, the experiment was immediately discontinued and the skin cooled.

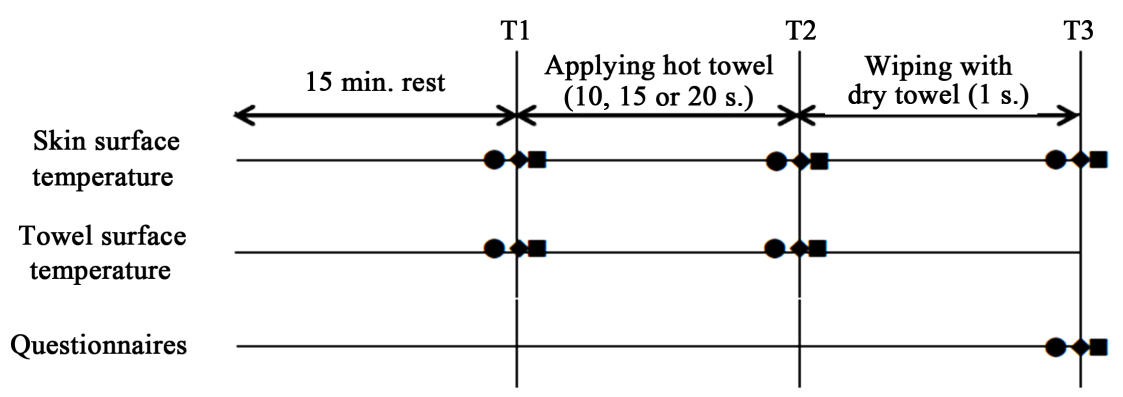

Figure 2. Study protocol in experiment A. AHT10s; $\bullet$ AHT15s; -, AHT20s; AHT, application of hot towel on the skin; T1, beginning of experiment; T2, shortly after applying a hot towel on the skin; T3, shortly after wiping with dry towel. 
Thermographic images were obtained using the Thermography R300 system (NEC, Tokyo, Japan; accuracy, $\pm 1^{\circ} \mathrm{C}$ ) at three times points (Figure 2): prior to the application (baseline skin, T1), shortly after application (exterior surface of the towel, T2) and immediately following wiping of the skin with a dry towel (interior surface of the towel and skin, T3).

The self-report questionnaire was completed after each condition, AHT 10-s, 15-s and 20-s. Participants rated warmth on a 4-point scale: "very warm", 4; "warm", 3; "cold", 2; and "very cold", 1. The assumption was that a rating of "very warm" would be reported immediately after towel application. The duration of hot towel application was rated on a 3-point scale: "perfect", 3; "I want to keep it on longer", 2; and "I want to remove it immediately", 1. The comfort level before removing the hot towel was rated on a 4-point scale: "very pleasant", 4; “a little pleasant”, 3; “a little unpleasant”, 2; and "unpleasant”, 1.

\subsection{Protocol for Experiment B}

Experiment B was conducted under the same conditions as for Experiment A, using a cross-over design for the presentation of the two conditions (bed bath with AHT 10-s and bed bath without AHT) randomizes a priori. For the AHT 10-s condition, the same cloth as in Experiment A was used, at the same temperature and with the same method of application. Again, if a participant complained of excessive skin heating, the experiment was immediately discontinued and the skin cooled. After removal of the hot towel, the skin of the back was dried with a towel, wiping the back in a cephalo-caudal direction, with 4 wipes repeated. The same wiping method was used for the condition without AHT.

The following thermographic images were obtained: surface skin temperature of the back, at baseline, after the 10-min rest period (T1); exterior surface of the towel immediately after application (T2); surface skin temperature of the back immediately after removal of the hot towel and 4 wipes for the AHT 10-s and the interior surface of the towel (T3); and after wiping only for the without AHT condition (T4).

The same self-report questionnaire regarding perceived warmth and comfort was completed after each condition, bed bath with AHT 10-s and bed bath without AHT (Figure 3).

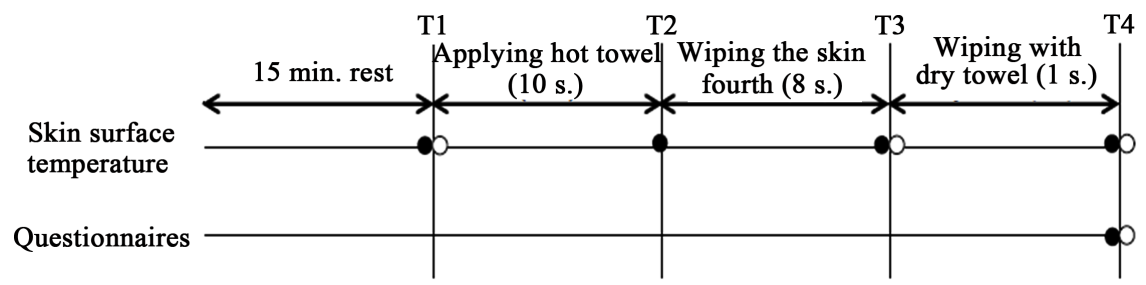

Figure 3. Study protocol in experiment B. Bed bath with AHT10s; O, Bed bath without AHT10s; AHT10s, application of hot towel on the skin for $10 \mathrm{~s}$; T1, beginning of experiment; T2, shortly after applying a hot towel on the skin; T3, shortly after wiping the skin thrice; T4, shortly after wiping with dry towel. 


\section{Statistical Analysis}

For experiment $\mathrm{A}$, skin and hot towel temperatures between the three conditions AHT 10-s, AHT 15-s and AHT 20-s, were compared using two-factor repeated-measure analysis of variance (ANOVA), with condition as a 3-level factor (AHT 10-s, AHT 15-s and AHT 20-s) and time as a 3-three level factor (T1, T2, and $\mathrm{T} 3$ ), including condition $\mathrm{X}$ time interactions. A single factor repeated measures ANOVA and multiple comparisons were performed when one factor or interaction was identified to be significant. Paired t-tests were performed for intra-subject comparison of temperatures across the three conditions.

For experiment B, the effect of condition (bed bath with AHT 10-s and bed bath without AHT) and time (T1, T2, T3, and T4) were again evaluated using a two factor repeated measures ANOVA, including condition $\times$ time interactions. A single factor repeated measures ANOVA and multiple comparisons were performed when one factor or interaction was identified to be significant. Paired t-tests were performed for intra-subject comparison of subjective evaluation between the two conditions (bed bath with AHT 10-s and bed bath without AHT 10-s).

All analyses were performed using InfReC Thermography Studio 5.1 advanced thermal images analysis software (Nippon Avionics, Tokyo, Japan) and SPSS (Version 22, IBM, Armonk, New York, NY, USA).

\section{Results}

\subsection{Experiment A}

Twenty participants completed all conditions of the experimental methods, 10 men and 10 women, with a mean age of $21.6 \pm 0.7$ years (range, 20 - 23 years) (Table 1). The baseline skin temperature (T1) was comparable for the 3 AHT conditions $(F=2.734, p=0.079)$ : AHT $10-\mathrm{s}, 33.6^{\circ} \mathrm{C} \pm 0.8^{\circ} \mathrm{C}$; AHT $15-\mathrm{s}, 33.8^{\circ} \mathrm{C} \pm$ $0.7^{\circ} \mathrm{C}$; and AHT $20-\mathrm{s}, 33.7^{\circ} \mathrm{C} \pm 0.6^{\circ} \mathrm{C}$. As well, the condition $\mathrm{X}$ time interaction was also non-significant $(F=2.734, p=0.079)$. However, there was a significant time-dependent difference in skin temperature for all three conditions $(F=$

Table 1. Subjects characteristics and implementation environment.

\begin{tabular}{|c|c|c|c|c|c|c|}
\hline & & \multicolumn{3}{|c|}{ Experiment A $(\mathrm{n}=20)$} & \multicolumn{2}{|c|}{ Experiment B $(n=21)$} \\
\hline & & $10-\mathrm{s}$ & $15-s$ & $20-s$ & $\begin{array}{l}\text { without } \\
\text { AHT10s }\end{array}$ & $\begin{array}{c}\text { with } \\
\text { AHT10s }\end{array}$ \\
\hline Age (SD) & & \multicolumn{3}{|c|}{$21.6(0.7)$} & \multicolumn{2}{|c|}{$21.7(0.8)$} \\
\hline \multirow[t]{2}{*}{ Sex } & Male & \multicolumn{3}{|c|}{$10(50.0 \%)$} & \multicolumn{2}{|c|}{$11(52.4 \%)$} \\
\hline & Fmale & \multicolumn{3}{|c|}{$10(50.0 \%)$} & \multicolumn{2}{|c|}{$10(47.6 \%)$} \\
\hline BMI (SD) & & & $20.7(2.0)$ & & \multicolumn{2}{|c|}{$21.2(2.1)$} \\
\hline \multirow{2}{*}{$\begin{array}{l}\text { Implementation } \\
\text { environment }(\mathrm{SD})\end{array}$} & Room temperature & \multicolumn{5}{|c|}{$24.8(0.7)$} \\
\hline & Humidity & \multicolumn{5}{|c|}{$60.2(5.8)$} \\
\hline
\end{tabular}

AHT10s, application of hot towel on the skin for 10 seconds. 
1383.282, $p<0.001$ ), with the mean surface temperature of the skin being higher at T2 and T3, compared to T1 ( $p<0.001$; Table 2$)$.

The mean surface temperature of the skin at T1 for the 3 conditions was as follows: AHT $10-\mathrm{s}, 45.3^{\circ} \mathrm{C} \pm 0.9^{\circ} \mathrm{C}$; AHT $15-\mathrm{s}, 45.4^{\circ} \mathrm{C} \pm 0.6^{\circ} \mathrm{C}$; and AHT 20-s, $45.1^{\circ} \mathrm{C} \pm 0.7^{\circ} \mathrm{C}$. Although the skin temperature was comparable between the 3 conditions at $\mathrm{T} 1$, the surface temperature of the towel was significantly different between conditions $(F=10.978, p<0.001)$ and across time $(F=5997.011, p<$ $0.001)$, with a significant condition $\mathrm{X}$ time interaction $(F=10.978, p<0.001)$. The mean hot towel surface temperature was significantly lower at T2 $(p<$ 0.001 ) than at $\mathrm{T} 1$ for each condition. A significant difference in towel temperature at T2 was also observed between conditions (Table 2): AHT 10-s and AHT 15 -s $(p=0.014)$; AHT 10-s and AHT 20-s $(p<0.001)$; and AHT 15-s and AHT 20 -s $(p<0.001)$.

With regard to the difference between the surface temperature of the skin and towel temperature (DST), at T2, there was a significant difference between the 3 conditions, as follows (Table 2): AHT 10-s and AHT 15-s $(p=0.001)$; AHT 10-s and AHT 20-s $(p<0.001)$; and AHT 15-s and AHT 20-s $(p<0.001)$.

Table 2. Comparison between bed bath without AHT10s and Bed bath with AHT10s regarding skin and towel surface temperature.

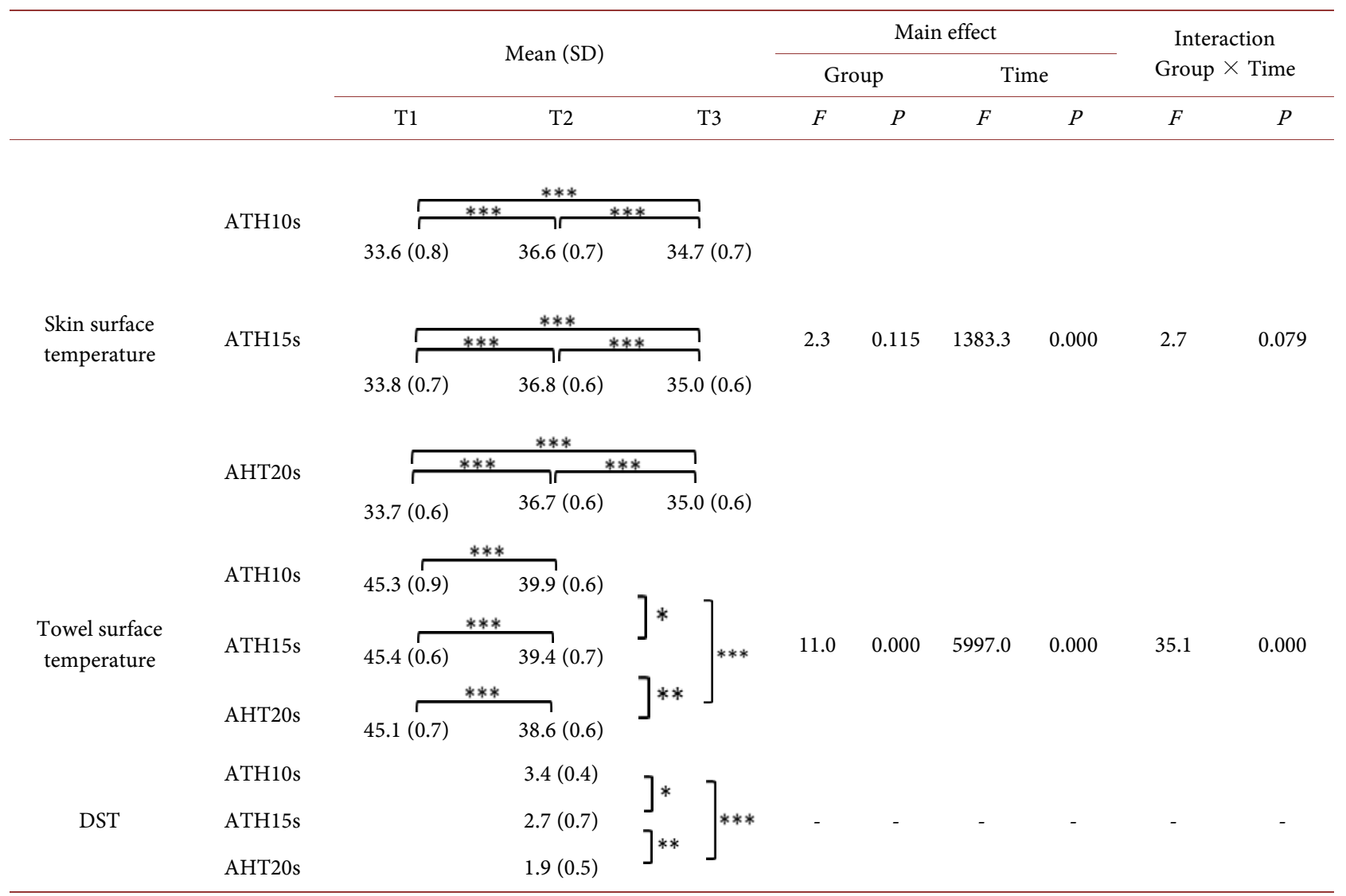

Two-factor repeated measure ANOVA. ${ }^{\star}$ Paired $t$-tests. ${ }^{* *} P<0.000,{ }^{* *} P<0.01,{ }^{*} P<0.05$. DST, difference between skin surface and towel temperature; AHT, application of hot towel on the skin; T1, beginning of experiment; T2, shortly after applying a hot towel on the skin; T3, shortly after wiping with dry towel. 
Subjective ratings were comparable between the 3 conditions for warmth ( $p=$ $0.171)$ and duration $(p=0.293)$. However, there was a significant difference in perceived comfort between AHT 10-s and AHT 20-s $(p=0.046)$, with an increase in duration of application (from 10-s to 15-s and 20-s) was associated with discomfort (Figure 4).

In summary, although there was no significant difference in skin temperature between the 3 conditions, comfort was most highly rated for AHT 10-s. This difference in perceived comfort was deemed to be associated with the DST, which was the largest for AHT 10-s at T2, indicating the greatest transfer of temperature from the towel to the skin. Therefore, 10-s is the most effective duration for applying a hot towel on the back.

\subsection{Experiment B}

Twenty-one participants completed all conditions of the experimental methods, 11 men and 10 women, with a mean age of $21.6 \pm 0.8$ years (range, 20 - 23 years) (Table 1). The mean surface skin temperature was comparable at $\mathrm{T} 1$ for both conditions, $33.9^{\circ} \mathrm{C} \pm 0.6^{\circ} \mathrm{C}$ for bed bath with AHT 10 -s and $33.7^{\circ} \mathrm{C} \pm 0.6^{\circ} \mathrm{C}$ for bed bath without AHT. A significant difference in surface skin temperature was identified across time $(F=64.790, p<0.001)$ and the 2 conditions $(F=459.014$, $p<0.001)$, with a significant condition $\mathrm{X}$ time interaction $(F=378.839, p<$ 0.001). Significant differences in skin temperature between conditions were identified at T3 and at T4 $(p<0.001)$. Moreover, the skin temperature was different across all pairings of time in the bed bath with AHT 10-s $(p<0.001)$, with the temperature being higher at $\mathrm{T} 1$ than at $\mathrm{T} 3$ and at $\mathrm{T} 4(p<0.001$; Figure 5$)$.

With regard to subjective ratings, both warmth $(p<0.001)$ and comfort $(p<$ 0.001) were higher for the bed bath AHT 10-s than bed bath without AHT (Table 3).

\section{Discussion}

\subsection{Effective Duration of Hot Towel Application to the Back}

In experiment $\mathrm{A}$, application of a hot towel to back increased the surface temperature of the skin to $34^{\circ} \mathrm{C}$ or higher, after dry wiping, in all 3 conditions (AHT10-, 15- and 20-s). According to Schmidt [12], an increase in the surface temperature of the skin of $0.3^{\circ} \mathrm{C}$ to $0.4^{\circ} \mathrm{C}$, from an initial temperature of $33^{\circ} \mathrm{C}$, is required for a noticeable increase in perceived warmth. In all 3 conditions, a

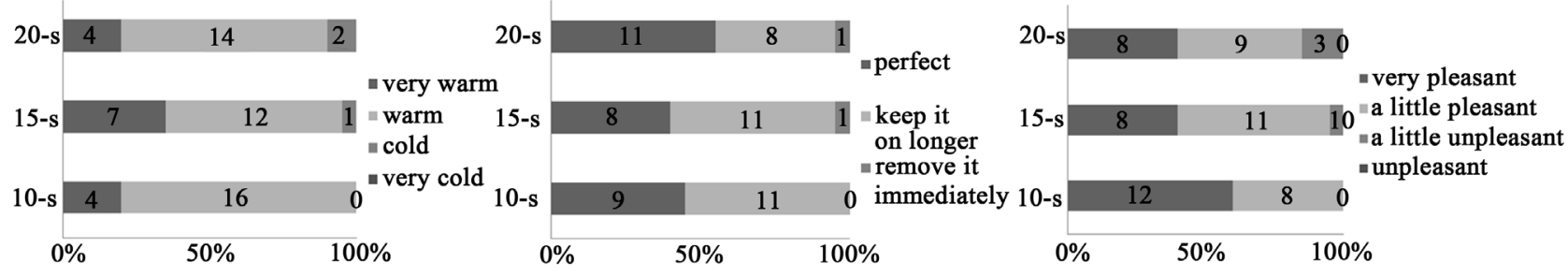

Figure 4. Comparison between Bed bath AHT10s, AHT15s and AHT20s regarding the subjective valuation AHT, application of hot towel on the skin. 


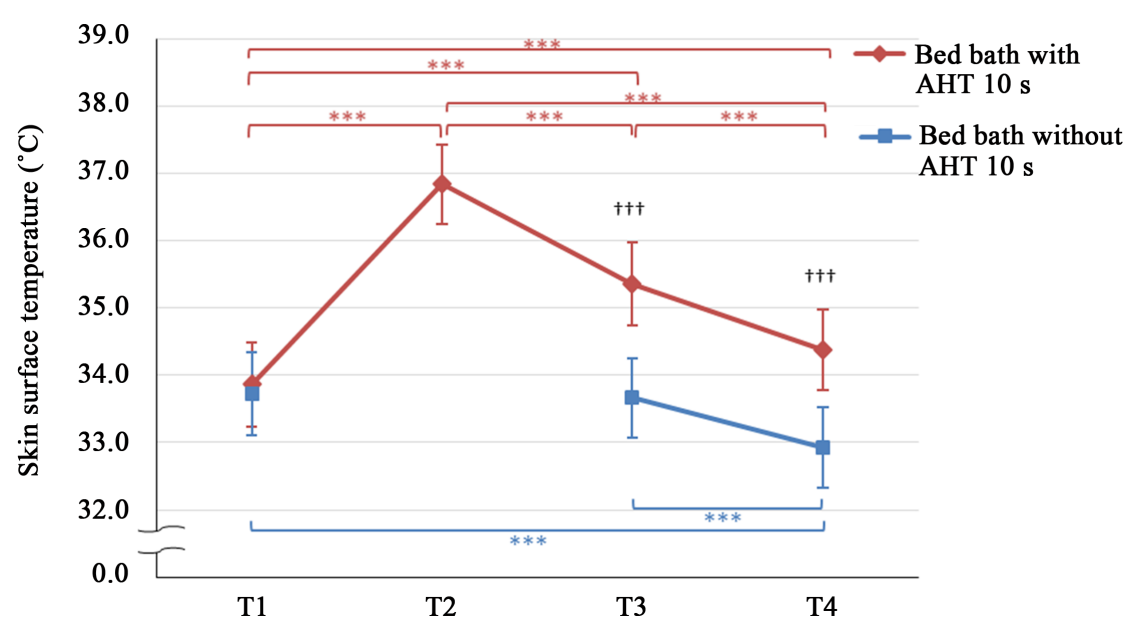

Figure 5. Time courses users of skin surface temperature. ${ }^{\dagger}$ Paired $t$-tests. ${ }^{\dagger \dagger \dagger} P<0.001$; T1, beginning of experiment; T2, shortly after applying a hot towel on the skin; T3, shortly after wiping the skin thrice; T4, shortly after wiping with dry towel; AHT10s, application of hot towel on the skin for 10 seconds.

Table 3. Time courses of skin surface temperature.

\begin{tabular}{|c|c|c|c|}
\hline & $\begin{array}{l}\text { Bed bath without AHT } 10 \mathrm{~s} \\
\qquad \mathrm{M}(\mathrm{SD})\end{array}$ & $\begin{array}{c}\text { Bed bath with AHT } 10 \mathrm{~s} \\
\text { M (SD) }\end{array}$ & $P$-value \\
\hline Warmth & $2.7(0.6)$ & $3.3(0.6)$ & 0.001 \\
\hline Comfort & $2.8(0.8)$ & $3.2(0.8)$ & 0.047 \\
\hline
\end{tabular}

${ }^{*}$ Paired $t$-test. ${ }^{\star} P<0.05,{ }^{*} P<0.01 . \mathrm{SD}$, standard deviation. AHT10s, application of hot towel on the skin for 10 seconds.

minimum increase of $1.1^{\circ} \mathrm{C}$ was observed, reaching the thermal sensation threshold for a perceived increase in warmth.

As a principle of heat transfer, heat moves from a warmer to a cooler area [13]. Therefore, heat from the towel will radiate to the ambient air, as well as to the skin in contact. In Experiment A, the mean surface temperature of the towel surface was significantly lower for AHT 20-s than AHT 10-s and 15-s, with the highest DST identified for the AHT 10-s condition. Therefore, as the duration of application increases, there is an increase in heat dissipation to the ambient air and application site, resulting in a decrease in the surface temperature of the towel, with the body adjusting to the decreasing DST with longer duration of hot towel application. Thus, the highest mean comfort rating was reported for the AHT 10-s, with responses of "a little unpleasant" for comfort and 'cold' for warmth reported for application durations of 15- and 20-s.

Based on our findings, a 10-s application of a hot towel to the back was the most suitable for achieving optimal comfort. 


\subsection{Efficacy of a 10-s Application of a Hot Towel to the Back}

The surface temperature of the skin significantly increased under the AHT 10-s condition, with an increase in temperature of $2.4^{\circ} \mathrm{C}-3.5^{\circ} \mathrm{C}$ achieved $(p<0.001)$. Moreover, the surface temperature of the skin on the back was significantly higher at T3 and T4 for bed baths with AHT 10-s compared to without AHT. After dry wiping (T4), the surface temperature was $0.5^{\circ} \mathrm{C}$ higher than at baseline for the AHT 10-s condition, but had decreased by $0.8^{\circ} \mathrm{C}$ for the condition without AHT. This decrease in surface skin temperature for dry wiping without AHT likely results from heat radiation from the surface of the skin to the ambient air with exposure. In Experiment B, therefore, subjective ratings of warmth and comfort were higher for bed bathing with than without AHT.

Based on our findings, a 10-s application of a hot towel to the back is more effective than bed bathing alone to increase skin temperature and comfort.

Our findings do not agree with those of Shishido and Yano [2] who did not report a significant difference in subjective comfort or increase in skin temperature for bed bathing with and without AHT application to the palmar surface of the forearm in elderly individuals. These differences between our findings and those of Shishido and Yano are likely to be related to the surface area of skin exposed to the hot towel. According to Tsukakoshi [14], calcitonin gene-related peptide-positive nerve fibers, which regulate blood flow to the skin, are widely distributed in the trapezius muscles, which are superficial muscles. Moreover, Iriki [15] reported that the strength the response in blood flow was inversely proportional to the area of skin stimulated. Accordingly, application of a hot towel on the back, which includes the trapezius muscles, would be more effective in increasing blood flow and raising the surface temperature of the skin than application to the palmar surface of the forearm.

Based on our findings, a 10-s application of a hot towel to the back, including the trapezius muscles, during bed bathing is effective in eliciting a sensation of warmth and improving comfort. However, in this study we have not measured the blood flow in the back, so we could not clarify the relationship between rising in skin surface temperature and comfort. Therefore, it is desirable to measure the blood flow at the back in the future.

\subsection{Study Limitations}

The anatomic location of the hot towel application was limited to the back, in this study; therefore, the effect of AHT10 s should be verified for other anatomic sites. Furthermore, it was targeted only for healthy adults; thus, the effect of AHT $10 \mathrm{~s}$ on hospitalized patients and the elderly should be considered.

\section{Conclusion}

A 10-s application of a hot towel to the back increased surface skin temperature and subjective comfort, and may provide advantages over traditional bed baths that do not include the application of a hot towel to the skin. 


\section{Acknowledgements}

The authors wish to acknowledge everyone who cooperated in the experiment. We thank the staff members of the Departments of Faculty of Health Sciences, Hokkaido University, for their assistance throughout this study.

\section{References}

[1] Massa, J. (2010) Improving Efficiency, Reducing Infection, and Enhancing Experience. British Journal of Nursing, 19, 1408-1414.

https://doi.org/10.12968/bjon.2010.19.22.1408

[2] Shishido, I. and Yano, R. (2017) Pilot Study on Benefits of Applying a Hot Towel for $10 \mathrm{~s}$ to the Skin of Elderly Nursing Home Residents During Bed Baths: Towards Safe and Comfortable Bed Baths. Geriatric Nursing, 38, 442-447. https://doi.org/10.1016/j.gerinurse.2017.02.008

[3] Tamura, T. (2017) A Study on the Difference of Temperature and Moist Sensation on the Skin Surface. Journal of Sensory Evaluation, 11, 81-88. (In Japanese)

[4] Kaneko, K. and Norimatsu, S. (2012) Comparison between the Relaxation Efficacy of the Moist-Heat Method and Dry-heat Method of Lower-Back Hot Fomentation. Journal of Japanese Society of Nursing Research, 35, 37-46. (In Japanese)

[5] Matsumura, C. (2004) Physical Effects of Thermal and Rubbing Stimuli: Comparison between Bed Bath Treatments and Hot Towel Baths. Kagawa Prefectural College of Health Sciences, 5, 1-10. (In Japanese)

[6] Okazaki, A., Abe, M., Uchiyama, M., et al. (2006) Comparison of Hot Towel Thickness Whipped Soap During Bed Bathing of the Back. Journal of Japanese Nursing Society: Nursing Comprehensive, 37, 188-190. (In Japanese)

[7] Shishido, I., Takeda, S., Hosokawa, Y., Iwakiri, N., Yoshida, Y. and Yano, R. (2015) Effects of Short-term Hot Towel Application to the Skin during Bed Bathing: Changes in Skin Surface Temperature, Stratum Corneum Water Content, ATP, and Subjective Evaluations. Japanese Journal of Nursing Art and Science, 14, 185-194. (In Japanese)

[8] Shishido, I. and Yano, R. (2016) Effective Time of Short-Term Hot Towel Application to Skin during Bed Bathing of the Elderly: Comparison of No Hot Towel Application during Bed Baths and Hot Towel Application for 7, 10 Seconds During Bed Baths. Japanese Journal of Nursing Art and Science, 15, 188-194. (In Japanese)

[9] Tsukakoshi, M., Funakoshi, K. and Hishinuma, N. (2014) Changes in Skin Temperature and Blood Flow in the Upper Body Following Application of a Hot Compress at $60^{\circ} \mathrm{C}$ to the Back. Japanese Journal of Nursing Art and Science, 13, 219-229. (In Japanese)

[10] Tagami, H. (2002) To Diagnose a Skin Disease in Equipment: Measure of the Function of the Stratum Corneum and of the Barrier Function. In: Tagami, H. and Talolawa, M., Eds., Dermatology Practice 14 Skin Clinics using the Apparatus, Bunkoudo, Tokyo, 2-5. (In Japanese)

[11] Kikuchi, K. and Tagami, H. (2002) To Diagnose a Skin Disease in Equipment: Measure the Function of the Stratum Corneum and of the Stratum Corneum Water. In: Tagami, H. and Talolawa, M., Eds., Dermatology Practice 14 Skin Clinics Using the Apparatus, Bunkoudo, Tokyo, 10-14. (In Japanese)

[12] Schmidt, R.F. (1981) Fundamentals of Sensory Physiology. Springer, New York. https://doi.org/10.1007/978-3-662-01128-7 
[13] Hishinuma, N. and Kawashima, M. (2013) Science and Verification of Nursing Art. Tokyo, Japan. Japanese Nursing Association Publishing Company, 114. (In Japanese)

[14] Tsukakoshi, M. (2008) Searching for Evidence When It Feels Good: Effects of Hot on the Back-A Search from a Neurological Anatomical Point of View of the Skin and Muscles. EB Nursing, 8, 438-445. (In Japanese)

[15] Iriki, M. (2003) Body Temperature Physiology Text. Bunkoudo, Tokyo, 98-100. (In Japanese) 